\title{
THE USE OF MOBILE AGENT BASED SYSTEM AS A SOLUTION FOR EFFICIENT MANAGEMENT OF TELECOMMUNICATION NETWORKS AND SERVICES IN A MULTI- DOMAIN ENVIRONMENT
}

\author{
Nazim Agoulmine ${ }^{1}$, José Neuman de Souza ${ }^{2}$, \\ Jorge Luis de Castro e Silva ${ }^{3}$, Arnoldo Nunes da Silva ${ }^{4}$ \\ ${ }^{\prime}$ Laboratoire PriSM, University of Versailles, E-mail: nazim.agoulmine@prism.uvsq.fr \\ ${ }^{2}$ Department of Computer Science,Federal University of Ceará,E-mail: neuman@ufc.br \\ ${ }^{3}$ Departament of Technology, University of Fortaleza(UNIFOR),E-mail: jlcsilva@ufc.br \\ ${ }^{4}$ Department of Informatics,Federal University of Pernambuco, E-mail: ans@di.ufpe.br
}

Key words: Management by delegation, Shared Management Knowledge, Co-operative Objets, Distributed Objects, Mobile Agents

Abstract: The liberation of telecommunication services provision worldwide will require an intensive co-operation between telecommunication operator to provide new types of end to end telecommunication services. However, the heterogeneity between service management systems is critical and makes an end to end service management very complex. Thus, it is important to define mechanisms that permit to hide this heterogeneity and permit the exchange of management information between domain and to distribute the control over various underlying sub management systems. This work analyses the use of mobile agents and co-operative remote objects as forms to implement the co-operative management model that permit delegation and co-operation between management domains. In fact, delegation paradigm and mobile agents technology provides a powerful framework that helps the interoperability between telecommunication management systems for the support of end to end service management.

The original version of this chapter was revised: The copyright line was incorrect. This has been corrected. The Erratum to this chapter is available at DOI: 10.1007/978-0-387-35581-8_35 


\section{INTRODUCTION}

The goal of this paper is to present a mobile agent based architecture to support multi-domain co-operation for the purpose of end to end management of telecommunication services. A special attention is given to the service trading mechanisms that allow value added provider to discover new basic service for the deployment of global advanced telecommunication services such as broadband virtual private networks service or TeleEducation service.

This work is mainly influenced by contemporary technological development from FIPA, OMG-MASIF, OSI (RM-ODP) [9], OMG (OMA, CORBA) [14], TINA-C [1], NM-Forum [11], Eurescom [21].

The idea of using mobile agent is motivated by the fact that centralised approach, such as OSI/CMIP [10] and IETF/SNMP [17] are not efficient enough when dealing with service level management. In the classical centralised approaches, management data are maintained by agents located in the managed devices and collected and analysed in a central way by a manager. The centralised approach for networks management has serious problems such as:

- The excessive traffic in the network between the manager and the agents and consequently the manager bottleneck. It involves an unnecessary amount of transmission between the manager and the agents in function of all the data being taken by the manager;

- The low performance of the network and the management system in function of the high traffic;

- The operator is overloaded with the amount of data and the number of alarms and events that request its attention;

- The lack of flexibility requested by the heterogeneous environment due to the increase of the services generated by the managements of the several networks (ATM and other).

The idea of this approach is to delegate management activities to cooperative management systems by mean of mobile agent. This approach was initially proposed for network management by mean of elastic agents and management by delegation paradigm [6][7]. The management by delegation can be seen as a mixture of the paradigm of the evaluation-remote [19] (where the delegated agents codes are sent to elastic servers) with the clientserver paradigm (where the manager controls the delegated agents execution remotely). Therefore, the manager can delegate the management tasks to processes located in another machines or in the machines that shelter the own managed resources. 
With the development of mobile agent technology, management by delegation found a framework where the distribution of control is naturally realised by mobile agent interactions.

Hence, the work undertaken by the OMG [16] through the standardisation of the architecture of object management, generally referenced by its main component: CORBA [14] provides the basic technology for protocol neutral interaction between management domains. The integration between CORBA and existing network management environments can be undertaken using proxy systems between SNMP and CORBA or CMIP and CORBA as defined by JIDM group (X/Open Joint Inter-Domain Management) [20].

Thus this article presents, as a proposal, an architecture, models and mechanisms that permits to deploy co-operation activities between telecommunication management systems for the purpose of end to end service management. The solution uses CORBA ORB (Object Request Broker) as the basic middleware to support the interaction between management system and the mobile agents as entities for the co-operation between heterogeneous telecommunication management domains.

\section{TMN FRAMEWORK}

A TMN system aims to respond to management needs concerning telecommunication networks and services management. A TMN provides management functions for these networks and services as well as the communication between TMNs. This management occurs by the exchange and the computation of information in order to assist the administrations in their role. An administration may comprise many TMNs and a TMN may as well cross many administrations. The TMN architecture allows the interoperability of networks managed separately too in order to provide inter-network services throw different administration.

TMNs interoperability and user's access to a given TMN are assumed at the service management layer where the service management OSFs (Open Systems Foundation). assume the responsibility of contractual aspects of services provided to clients as well as interfacing with other administrations and services interactions.

A set of reference points delimiting the managing function blocs inside and at the boundary and identifying the management information moving between blocs are identified by the TMN architecture [22]. The interface placed between two OSF blocs belonging to two different TMNs inside the same or in different administration domains is symbolised by the $\mathrm{X}$ interface. 
In the actual deregulation of telecommunication, the deployment of the service and service management systems is intended to support the multiprovider and multi-customer service provision scenario. Thus, TMNs may co-operate to provide a global and end-to-end service management from the customer viewpoint. Hence, there is a need to deploy open service to support the Open Service Market, where the VASP (Value Added Service Provider) can discover, combine and enhance existing management services to target particular markets.

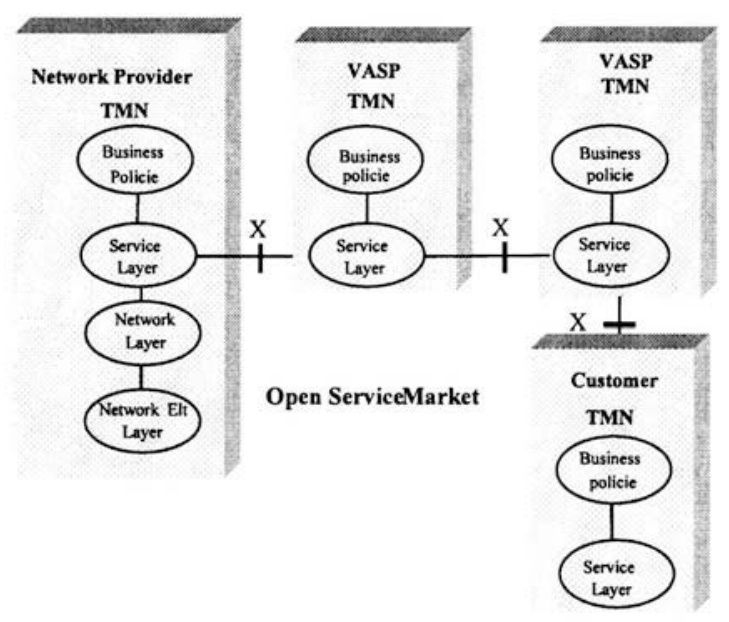

Figure 1. Interactions between Customer, VASP, PNO TMNs

Thus to support these different interaction, it is necessary to identify exactly the interfaces between the TMN at the service management layer. The information exchanged between TMNs and network users is exclusively related to TMN management functions in order to offer information concerning the usage done by these users on behalf of the network as well as a limited level of command supposing certainly that service providers supply management services to users. A common access to services and networks management application has to be provided to users in quit some conditions of security and access rules and respecting shared management schema.

\section{MOBILE AGENTS}

Agent technology is a growing area of research and new application development in telecommunications. Having highlighted the main requirements for security management, the intelligent agent concept seems to be a candidate approach to fulfil these requirements. What is an Agent? 
Until now, there is no an internationally accepted definition of an intelligent agent concept [15][19]. The term Agent is a concept used in different area and having different meaning depending on the context. Nevertheless, different types of agents reflect a set of properties, which common among them and are described below:

- Autonomy: is the ability of an agent to operate without direct intervention of humans or other agents and to have some kind of control based on its internal and/or external environments;

- Co-operation: an Agent is co-operative and is able to have a social ability. This sociability allows an agent to interact with other agents for the purpose of performing tasks that are beyond the capability of a particular agent. This capability goes from delegation (distribution of sub-tasks) to peer-to-peer inter-working;

- Pro-activeness: it is the agent's ability to anticipate situations and change its course of action to avoid them. Proactive agents are capable of exhibiting goal-direct behaviours by taking some initiative;

- Reactivity: this kind of behaviour means that the agent reacts in realtime to changes that occur in its environments;

- Adaptability: is the ability of an agent to modify its behaviour over time to fulfil its problem-solving goal;

- Intelligence: the term "Intelligence" means that the agent is able to exhibit a certain level of intelligence priority, ranging from predefined actions (planning) up to self learning (define new actions);

- Flexibility: is the ability an agent should have to adapt itself to cope with the environment in which it is situated;

- Mobility: an Agent is mobile. It is capable of moving from one location to another in order to perform a particular task or to react to a particular event.

There are good reasons to use mobile agents (MA) in network management applications. In fact, mobile agents reduce the management traffic, because they pack a conversation among objects and they travel with the package to a target host where the interactions can occur locally. They permit to optimise the execution of management tasks as they operate in an asynchronous form and are autonomous. Thus it is possible to execute a number of tasks in the same time. Hence, they permit to hide the heterogeneous of execution environment by means of mobile agent execution environments.

Hence, they are dynamically adaptive in the sense that it is possible to customise the behaviour of mobile agents depending of the target environment. 


\section{DELEGATION MANAGEMENT THROUGH THE $\mathrm{X}$ INTERFACE}

The $\mathrm{X}$ service interface aims to provide to management application in one TMN domain a service view of other TMN domains. The idea in this paper is not use the classical Manager/Agent interaction at this interface but rather the delegation paradigm.

In fact, in the classical manager/agent mode, the agent don't have any autonomy (expect for notifications) and is statically in term of functionality. If this approach can be useful at the network element level, it is very useless at the service layer where interaction between TMN is performed.

At the service level, it is very interesting to delegate some responsibilities to other processes distributed in the same or remote TMN. Some tasks, inside of the model, can still be controlled by the central authority, but in many cases the local managers (delegated processes) can execute several tasks in an autonomous way [21].

Mobile agents provide the paradigm for the delegation of management activities.

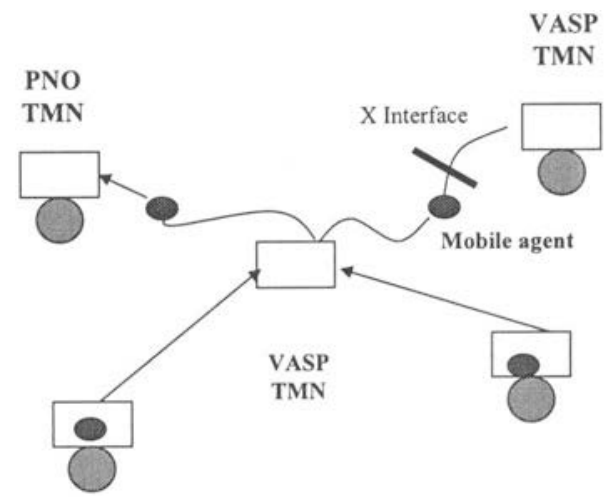

Figure 2. Co-operation by delegation

The MbD model, in contrast with the approach of centralised management, supports management model scalability. Among its advantages the following may be mentioned:

- The automation of the management functions by delegated mobile reduces the load in the operation centres;

- It eliminates or reduces significantly the need of constant polling in the network between the manager and the agents, as the mobile agent can perform directly the polling activities inside the target domain;

- New management services can be added in a dynamic way by a mean of new mobile agent to send. 
Nevertheless, management policies should be defined in order to define the authority to delegate management tasks to other TMN and to access particular management information. Each TMN should know what are its relationships with other TMN as well as the tasks it can delegate or perform.

\section{ABSTRACT BASIC MANAGEMENT SERVICE}

In order to support the delegation, a concept called Abstract Basic Management Service Building Block is introduced in the service management layer of the TMN. The idea behind this concept is to help to introduce the necessary abstraction at the service layer in order to hide to the service manager, developer and user the underlying complexity in term of information modelling, management protocol and service and network and system technologies. Thus, the focus at this layer will only be concerned by the service itself in terms of service modelling, service combination, service configuration, control and deployment in order to deploy quickly new enhanced services based on the interoperability of basic management service located in different TMN domains.

This concept is close to the Service Independent Building Blocks that is defined in the Intelligent Network architecture. The SIB are defined at the Global Functional Plane where the network is viewed as a single virtual machine upon which SIBs can be combined in a Global Service Logic to build an intelligent service that acts directly on the virtual machine.

The objectives is to render completely abstract to the service layer at the VASP TMN the particular specification and implementation of particular basic service in the NO (Network Operator) and Customer TMN. This approach is mandatory to define a global Open Service Market where service interaction and interoperability will be performed in an open and integrated manner.

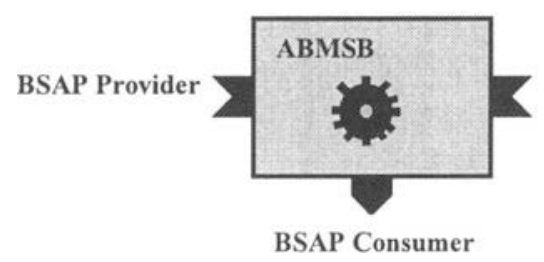

ABMSB : Abstract Basic Management Service Block BSAP : Basic Service Access Point

Figure 3. Abstract Basic Management Service Block 
In this approach, the organisation that would like to participate to the Open Service Market should specify the management services that would like to sell to the other participants in the form of ABMSB. Thus VASP TMN will re-use existing basic service existing at the PNO (Private Network Operator) TMN and or other VASP TMN to build rapidly new enhanced management service to satisfy specific customer's demands or to develop a new type of ABMSB that could proposed to other VASP as presented in the following figure:

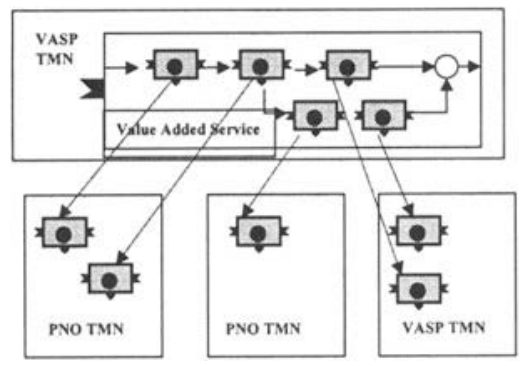

Figure 4. Basic Services Recombine and extend

This approach permits the rapid interoperability of service among a number of TMN without putting any constraints about the specification or the implementation of services inside TMN.

A particular service is built based on an interaction schema that specifies the logic of interaction between ABMSB for the purpose of an enhanced management service.

The Mobile agents provide the underlying techniques to support the effective use of ABMSB. The interactions between TMN are performed by means of mobile agent behaviour and mobile agent interaction. Mobile agent will migrate from one TMN to another to realise the physical interaction with the ABMSB. Each TMN define a set of functionality's that a mobile agent will have access in term of ABMSB in the local TMN mobile agent execution environment. The mobile agent constitutes the basic entities for component interaction and re-use. Using mobile agent, it permits to define in complete abstract manner the overall service while the realisation of the service is perfumed physically by agent transfer and agent remote activities. It is then possible to parallelise tasks, to define alternative schema and so on.

The ABMSM available at each TMN boundary can of general purpose (e.g. configuration, alarms, performance, accounting, security) or specialised on which can be discovered dynamically. 


\section{ENGINEERING ARCHITECTURE}

The engineering architecture defines the engineering components to deploy in order to support the previous concepts. The fundamental condition to execute an application that uses mobile code is an infrastructure Thus in order to support interoperability, each TMN that participate to the cooperation should provide such an infrastructure. This infrastructure provides services in the transport layer that support the execution and the movement of mobile codes. Such an example of execution infrastructure is Voyager vrs.2.0.0 platform developed by Object Space company. This platform supports mobile codes based on Java. But the infrastructure can also be based on any other environment as far as it provides the basic functionality's for agent mobility, interaction, security, etc.

On the top on this infrastructure, it is necessary to develop a set of services that permit to a running agent to interact with the existing ABMSB. ABMSB can encapsulate OSI or SNMP agent to retrieve or set management information as presented in the following figure.

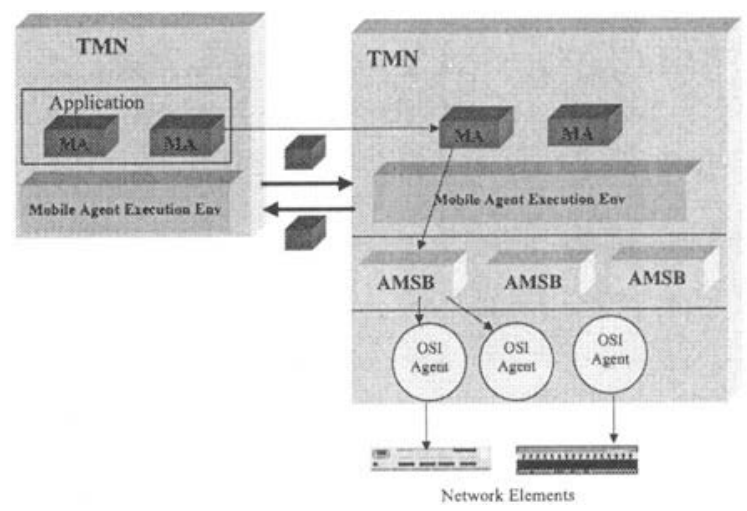

Figure 5. Mobile Agents base TMN Interoperability

But ABMSB can encapsulate more sophisticated functionalities such as VPN services, Security services, etc.

Thus, mobile agent sent to remote location has to have a minimum of knowledge and skill that will permit it to interact with the modelled remote environment in term of ABMSB. However, the interface with ABMSB is specified in term of BSAP (Basic Service Access Point), that permits to agent to discover dynamically all the services available at the particular ABMs. 


\section{CONCLUSION}

We have presented in this paper architecture for service interoperability in a context of multi-domains TMN. The architecture permits to different TMN to co-operate by means of mobile agent interaction and Abstract Basic Management Service Blocks. ABSMSB provides the basic entity that can be reused to build an end to end service management system. And the Mobile Agent technology permits to realise the delegation of management activities in remote management systems to order to render more efficient the overall management process.

An engineering architecture was defined and a prototype is being developed using HP OpenView Java Management API and Voyager.

Hence further studies are currently undertaken in order to enhance the mobile agent interaction model and the ABMSB specifications in order to clarify the definition of service and the way they can combined in order to provide rapidly and efficiently new enhanced services.

\section{REFERENCES}

[ 1] Barr, W., Boyd, T. and Inoue, Y.: "The TINA Initiative". In IEEE Communications Magazine, 31(3):70-76, 1993.

[ 2] Castro e Silva, J., Silva, A. N. and Souza, J. N.; "Applying Cooperative Remote Objects and Mobile Agents Concepts for Managing by Delegation Heterogeneous Network Systems". Proceeding of the IEEE ICT'99 - International Conference Telecommunications, Cheju, Korea, June 1999.

[ 3] Bieszczad, A. and Pagurek B.: "Application-Oriented Taxonomy of Mobile Code". Proceedings of the EEE/IFIP Network Operations and Management Symposium NOMS'98, New Orleans, Louisiana, February 1998.

[ 4] Borland: "Visibroker for Java 3.0 - Programmer's Guide", URL http://www.inprose.com/techpubs/

[ 5] Castro e Silva, J., Silva, A. N. and Souza, J. N.: "Designing and Developing Distributed Applications within the CORBA and Java Environments: Practical Issues and Critical Analysis", Proceeding of the SCI'98 and ISAS'98, Orlando - Flórida, July 1998.

[ 6] Goldszmidt, G. and Yemini Y.: "Distributed Management by Delegation", PhD Thesis, Columbia University, New York, NY, USA, December 1995.

[ 7] Yemini, Y., Goldszmidt, G. and Yemini, S.: "Network Management by Delegation", Proceeding of the IFIP $2^{\text {nd }}$ ISINM'91, Washington, D.C., USA, April 1991.

[ 8] Gosling, J. and McGilton, H.: “The Java Language Environment”, Sun Microsystems, Mountainview, CA 1995.

[ 9] ITU-T Rec. X.901 | ISO/IEC 10746 Information Technology - Open Distributed Processing - Reference Model (RM-ODP), 1996.

[10] ITU-T Recommendation X.701: "Information Technology - Open Systems Interconnection - System Management Overview", 1992.

[11] NM-Forum - Network Management Forum, URL http://www.nmf.org 
[12] Eurescom - European Institute for Research and Studies in Telecommunications, URL http://www.eurescom.de

[13] ObjectSpace: "ObjectSpace Voyager Core Package Technical Overview", URL http://www.objectspace.com/voyager/VoyagerTechOviewNEWTAGLINE.pdf, Dallas, USA, 1997.

[14] OMG: "The Common Object Request Broker: Architecture and Specification Ver. 2.0, Framingham Mass., July 1995.

[15] Pham,V. and Karmouch, A. : "Mobile Software Agents: An Overview”. IEEE Communications, Vol.36, No.7, pp.26-36, Special Issue on Mobile Agents and Telecommunications, 1998.

[16] Siegel, J.:"CORBA Fundamentals and Programming”. Wiley, New York, USA, 1996.

[17] Stallings, W.: "SNMP, SNMPv2 and CMIP: The Practical Guide to Network Management", Addison Wesley,1993.

[18] Sun Microsystems, Inc.: "Java Platform Documentation", URL http://java.sun.com/docs/index.html

[19] Vigna, G.:"Mobile Code Technologies, Paradigms, and Applications”. PhD Thesis, Politecnico di Milano, Milan, Itália, 1997.

[20] X/Open: "Inter Domain Management: Specification Translation". The Open Group ISNM 1-85912-150-0, February 1997.

[21] ITU-T Rec. X.901 | ISO/IEC 10746 Information Technology - Open Distributed Processing - Reference Model (RM-ODP), 1996.

[22] ITU-T Rec. M.3010 | Principles for a Telecommunications Management Network (TMN), Study Group IV, 1996. 